\title{
Ex situ Improvement of Indigenous Chicken in Bangladesh
}

\author{
S. Faruque, M.N. Islam ${ }^{1}$ and A.K.F.H. Bhuiyan ${ }^{2 *}$ \\ Poultry Production Research Division \\ Bangladesh Livestock Research Institute \\ Savar, Dhaka, Bangladesh
}

\begin{abstract}
This work is part of a long-term selection program carried out (i) to assess the performance of three indigenous chicken genotypes under intensive management and (ii) to evaluate the response to selection of three indigenous chicken genotypes. A total of 5945 day-old chicks representing three types of indigenous chicken genotypes namely Naked Neck $(N N)$, Hilly $(H)$ and Non-descript Deshi $(N D)$ were hatched for foundation generation $\left(G_{0}\right)$, first generation $\left(G_{1}\right)$, second generation $\left(G_{2}\right)$ and third generation $\left(G_{3}\right)$ for this study. In every generation, selection was practiced at 40-week of age on the basis of an index comprising the parameters of age at first egg (AFE), body weight $(B W)$, egg production (EP) and egg weight $(E W)$. The average body weights of $N D, H$ and $N N$ were 1074 \pm 4.0 , $1279.6 \pm 5.5$ and $1041.0 \pm 5.7 \mathrm{~g}$, respectively at the age of 16 weeks. The mean daily weight gain of indigenous chicken at 0-8, 0-12, 0-16 week and 0-maturity were 7.8 $\pm 0.02,9.4 \pm 0.02$, $9.8 \pm 0.02$ and $8.2 \pm 0.03 \mathrm{~g}$, respectively. The lowest hatchability was found in NN genotype (77.5\%). The average egg production of $N D, H$ and $N N$ were $83.9 \pm 1.1,76.2 \pm 1.4$ and $74.0 \pm 1.5$, respectively. Among the three indigenous chicken types, ND had significantly higher $(p<0.001)$ average number of eggs for a given period than $H$ and $N N$ genotypes. In terms of body weight, $H$ genotype was superior. Based on the performance of the four generations, the study revealed that $H$ genotype to be a good base population to be developed as a meet producing strain whereas non-descript Deshi chicken could be improved for.
\end{abstract}

Keywords: Economic traits, ex situ selection, indigenous chicken

\section{INTRODUCTION}

The improvement of productivity of indigenous chicken is a long felt requirement in Bangladesh. Indigenous chickens of Bangladesh are categorized as Non-descriptive Deshi (ND), Naked Neck (NN), Hilly (H), Aseel (AS) and Jungle fowl (Bhuiyan et al., 2005) in respect of the morphological variations as well as production performances. Bangladesh has a rich heritage of indigenous chicken germplasm, which strongly supported decisive measures for conserving indigenous genetic resources. Depending on the phenotype Bhuiyan et al. (2009) have predicted that indigenous chickens are genetically diluted in about $60 \%$. Indiscriminate random mating among indigenous types of chicken and unplanned crossing with exotic breeds are the main contributors for genetic erosion leading to loss of original characteristics of indigenous chicken. Hence, a valuable genetic

\footnotetext{
1 Department of Animal Breeding and Genetics, Bangladesh Agricultural University, Mymensingh, Bangladesh Corresponding author: bhuiyanbau@gmail.com
} 
characteristics may be lost forever from the indigenous chicken types. Conservation and preservation of resources as an insurance against future needs has become a topic of mounting concern (Crawford, 1984). The genetic potential of indigenous chicken in Bangladesh is poor. Their productivity is low and as a result they are unable to meet the demand for eggs and meat in the country. This is considered as an important constrain to poultry development, which could be overcome through genetic improvement of indigenous stock by appropriate breeding. Since no selection has been done except natural selection, there must be considerable genetic variance for various traits among them which may not be found in the exotic stock and hence, there is a scope for improvement through selective breeding. Therefore, the indigenous chicken must be conserved in adequate quantities for future use and for their improvement; selection and pure breeding is necessary.

Selection for egg and meat production of Genetic improvement of indigenous chickens has been carried out in many places of the world and it is evident that considerable improvement is possible (Bhuiyan et al., 2005), for example, in Bangladesh a number of studies on some productive and reproductive traits of Non-descript Deshi (ND), Hilly (H) and Naked Neck (NN) have been carried out by several researchers (Huque, 1999; Khatun et al., 2005; Bhuiyan et al., 2005; Faruque et al., 2007; Faruque et al., 2011). However, no systematic study has yet been carried out with planned selection and breeding of indigenous chickens under controlled conditions. Therefore, in order to improve the productivity of indigenous chickens the present work was undertaken to fulfil two objectives; to assess the productive and reproductive performances of three indigenous chicken genotypes under intensive management selected over three generations and to predict responses to selection for improving three indigenous chicken genotypes.

\section{METHODOLOGY}

\section{Formation of foundation stock}

As a part of selection and improvement of indigenous chicken, the Poultry Production Research Division (PPRD) of Bangladesh Livestock Research Institute (BLRI) to date has collected 5 types of indigenous chicken for its conservation and breeding. Selection of those five types has been done using phenotypic characteristics in the past breeding program. Foundation stock was established utilizing those existing stock of BLRI as well as by incorporating variation through screening of males/females/eggs from wide range of indigenous chicken gene pool in Bangladesh.

\section{Feeding and vaccination}

Concentrate mixtures that contain $20.1 \%$ crude protein and $2908 \mathrm{Kcal} \mathrm{ME} / \mathrm{kg} \mathrm{DM} ; 18.1 \%$ Crude Protein and $2904 \mathrm{Kcal} \mathrm{ME} / \mathrm{kg} \mathrm{DM}$ and $16.3 \%$ Crude Protein and $2845 \mathrm{Kcal} \mathrm{ME} / \mathrm{kg}$ DM were provided twice daily in the morning and evening during brooding, growing and laying periods, respectively. Fresh, cool and clean drinking water was made available for all the times. All chicks were vaccinated as per schedule given by veterinarian.

\section{Egg production traits}

Egg production traits were comprised of hen-day egg production, hen-house egg production and egg production rate, and calculated using Equation (i) to (iii), respectively. 


$$
\begin{aligned}
& \text { Hen day egg production }(\%)=\frac{\text { No. of eggs laid }}{\text { No. of hens at lay }} \times 100 \\
& \text { Hen house egg production }(\%)= \\
& \text { Egg production rate }(\%)=\frac{\text { No. of eggs laid }}{\text { No. of hen housed }} \times 100 \\
& \text { No. of hen days at lay }
\end{aligned}
$$

In addition 242 day and 315 day egg production calculated for all the birds.

\section{Fertility}

The fertility was obtained as percentage by using formula (iv).

Fertility $(\%)=\frac{\text { No. of fertile eggs }}{\text { Number of egg set }} \times 100$

\section{Selection objective}

The selection objectives of the study were to improve the egg production and / or growth rate of indigenous chicken belong to ND, $\mathrm{H}$ and $\mathrm{NN}$ genotypes. Improvement targets were set to increase egg weight by $1 \mathrm{~g}$, to increase egg production rate by $2 \%$ per generation, and to improve 8 - weeks body weight of ND, H, and NN chickens $(342,375$ and $331 \mathrm{~g}$, respectively) to $500 \mathrm{~g}$ after three generations of selection and breeding.

\section{Selection criteria}

In foundation generation and in $1^{\text {st }}$ and $2^{\text {nd }}$ generation, selection was practiced at 2 (two) stages; breeding value for 8 -week body weight and index value at 40 -weeks of age, where index comprises of age at first lay (days), body weight $(\mathrm{g})$ at 40 weeks of age, egg production rate (\%) at 168-280 days and egg weight (g) at 40 weeks of age.

The Selection Index (I) proposed and developed by Hazel (1943) was computed by the Equation (v).

Selection Index $(I)=b_{1} x_{1}+b_{2} x_{2}+\ldots \ldots \ldots \ldots+b_{n} x_{n}$

where, $\mathrm{x}_{1}, \mathrm{x}_{2}, \ldots \ldots . \mathrm{x}_{\mathrm{n}}$ represent the phenotypic values for the trait

$b_{1}, b_{2}, \ldots \ldots . b_{n}$ denote the relative weights given to each of the trait

\section{Mating design}

Selected males and females were mated assertively with a maximum male:female ratio of 1:5 using artificial insemination avoiding mating among close relatives in all the generation. 


\section{Statistical analysis}

Since sample size varied among three genotypes, data were analyzed based on nonorthogonal factorial experiment and using General Linear Model (GLM) procedure. SPSS 11.5 for Windows (SPSS, 1998) was used for data analysis. For all statistical purposes the theory of Snedecor and Cochran (1989) was followed. Duncan's multiple range test (DMRT) by Kramer (1957) was used for mean comparisons.

\section{Prediction of expected selection response $(\mathbf{R})$}

Expected genetic progress due to selection in a generation for egg production, egg weight, body weight and age at sexual maturity was estimated for $G_{0}, G_{1}$ and $G_{2}$ using Equation (vi) by Falconer (1981).

$$
\begin{aligned}
& R=1 / 2 h^{2} \times S_{f} \\
& \text { where, } h^{2}=\text { heritability of EP, EW, AFE and BW } \\
& S_{f}=\text { selection differential for the selected females }
\end{aligned}
$$

The selection response for 8-week body weight was calculated using Equation (vii).

$$
\mathrm{R}=\mathrm{h}^{2} \times \mathrm{S}
$$

where, $\mathrm{h}^{2}=$ heritability of 8 -week body weight and $\mathrm{S}=$ selection differential.

\section{RESULTS AND DISCUSSION}

\section{Body weight and weight gain}

Male chicks were significantly heavier $(\mathrm{p}<0.001)$ in body weights at $8^{\text {th }}, 12^{\text {th }}$ and $16^{\text {th }}$ weeks than the females. Many avian species, like chickens, showed marked dimorphism in body weight with males being substantially heavier than females. This could be due to the effect of male growth hormones (Singh et al. 1982). Significantly highest $(\mathrm{p}<0.001)$ body weights were observed in $\mathrm{G}_{3}$ generation compared to other generations in all stages. Significant body weight differences among the genotypes were observed at the age of sexual maturity, with the highest body weight observed for H $(1499.0 \pm 9.5 \mathrm{~g})$ genotype. Khandoker (1993) observed that body weight of indigenous chickens at 8,12 and 16 weeks of age averaged $186.5,475.0$ and $833.2 \mathrm{~g}$, respectively. Those weights recorded were much lower than the present findings. Faruque et al. (2007) reported heavier body weight at sexual maturity in $\mathrm{H}$ $(1461.2 \pm 251.0 \mathrm{~g})$ and the lowest weight in $\mathrm{NN}(1310.5 \pm 136 \mathrm{~g})$, recording more or less similar finding to the present study (Table 1).

The mean daily weight gain of indigenous chickens at $0-8,0-12,0-16$ week and 0 to maturity were $7.8 \pm 0.02,9.4 \pm 0.02,9.8 \pm 0.02$ and $8.2 \pm 0.03 \mathrm{~g}$, respectively (Table 2). Significantly highest daily gains were observed in $\mathrm{G}_{3}$ generation compared to other generations in all stages. The mean growth rate varied between sexes and between the times of measurement (Table 2). The mean growth rate in $16^{\text {th }}$ week was higher compared to what was observed in earlier studies (Wilson et al. 1987). Faruque et al. (2007) observed that daily body weight gain of 5.88, 6.17 and 6.27 g per bird at 8 weeks growth, respectively for ND, H and NN genotype under intensive management, and those results were less than the body weights recorded in the present study. Halima (2007) reported daily body weight gain from 5-8 
weeks of age ranged from $8.8 \mathrm{~g}$ in Gaassay chicken to $11.50 \mathrm{~g}$ in the Mecha chicken which was much higher than the findings in the present study.

Table 1. Variation of body weight (least square mean \pm standard error) in different sexes, genotypes and generation of selection

\begin{tabular}{|c|c|c|c|c|c|}
\hline Factor & Level & $\begin{array}{c}8^{\text {th }} \text { week } \\
\text { weight } \\
\text { (g) }\end{array}$ & $\begin{array}{c}12^{\text {th }} \text { week } \\
\text { weight } \\
\text { (g) }\end{array}$ & $\begin{array}{c}1^{16^{\text {th }}} \text { week } \\
\text { weight } \\
\text { (g) }\end{array}$ & $\begin{array}{l}\text { Weight at } \\
\text { maturity } \\
\text { (g) }\end{array}$ \\
\hline \multirow[t]{2}{*}{ Sex } & Male & $\begin{array}{c}507.4^{\mathrm{a}} \pm 1.6 \\
(2551)\end{array}$ & $\begin{array}{c}935.1^{\mathrm{a}} \pm 3.7 \\
(1249)\end{array}$ & $\begin{array}{c}1313.0^{\mathrm{a}} \pm 5.2 \\
(854)\end{array}$ & - \\
\hline & Female & $\begin{array}{c}425.3^{\mathrm{b}} \pm 1.5 \\
(2891)\end{array}$ & $\begin{array}{c}716.7^{\mathrm{b}} \pm 2.5 \\
(2060)\end{array}$ & $\begin{array}{c}954.9^{\mathrm{b}} \pm 3.2 \\
(1875)\end{array}$ & - \\
\hline \multirow[t]{3}{*}{ Genotype } & ND & $\begin{array}{l}441.6^{\mathrm{b}} \pm 1.4 \\
\quad(2910)\end{array}$ & $\begin{array}{c}776.8^{\mathrm{b}} \pm 2.8 \\
(1633)\end{array}$ & $\begin{array}{c}1074.6^{\mathrm{b}} \pm 4.0 \\
(1313)\end{array}$ & $\begin{array}{c}1212.2^{\mathrm{b}} \pm 6.6 \\
\quad(614)\end{array}$ \\
\hline & $\mathrm{H}$ & $\begin{array}{c}513.0^{\mathrm{a}} \pm 2.0 \\
(1363)\end{array}$ & $\begin{array}{c}920.2^{\mathrm{a}} \pm 3.9 \\
(886)\end{array}$ & $\begin{array}{c}1279.6^{\mathrm{a}} \pm 5.5 \\
(728)\end{array}$ & $\begin{array}{c}1499.0 \mathrm{a} \pm 9.5 \\
(308)\end{array}$ \\
\hline & NN & $\begin{array}{l}441.6^{\mathrm{b}} \pm 2.2 \\
\quad(1169)\end{array}$ & $\begin{array}{l}775.2^{\mathrm{b}} \pm 4.2 \\
\quad(790)\end{array}$ & $\begin{array}{c}1041.0^{c} \pm 5.7 \\
(688)\end{array}$ & $\begin{array}{c}1180.7^{\mathrm{c}} \pm 9.3 \\
(340)\end{array}$ \\
\hline \multirow[t]{4}{*}{ Generation } & $\mathrm{G}_{0}$ & $\begin{array}{c}356.6^{\mathrm{c}} \pm 2.2 \\
(1447)\end{array}$ & $\begin{array}{c}768.9^{c} \pm 4.8 \\
(662)\end{array}$ & $\begin{array}{c}1039.9^{\mathrm{d}} \pm 6.0 \\
(660)\end{array}$ & $\begin{array}{c}1221.5^{\mathrm{c}} \pm 8.0 \\
(429)\end{array}$ \\
\hline & $\mathrm{G}_{1}$ & $\begin{array}{c}468.9^{\mathrm{b}} \pm 2.2 \\
\quad(1328)\end{array}$ & $\begin{array}{l}803.1^{\mathrm{b}} \pm 4.5 \\
\quad(962)\end{array}$ & $\begin{array}{c}1121.9^{c} \pm 6.8 \\
(610)\end{array}$ & $\begin{array}{c}1355.7^{\mathrm{a}} \pm 8.8 \\
\quad(421)\end{array}$ \\
\hline & $\mathrm{G}_{2}$ & $\begin{array}{l}490.4^{\mathrm{b}} \pm 2.3 \\
\quad(1571)\end{array}$ & $\begin{array}{l}816.4^{\mathrm{b}} \pm 4.3 \\
\quad(795)\end{array}$ & $\begin{array}{c}1152.9^{\mathrm{b}} \pm 6.0 \\
(703)\end{array}$ & $\begin{array}{c}1309.3^{\mathrm{b}} \pm 9.2 \\
(412)\end{array}$ \\
\hline & $\mathrm{G}_{3}$ & $\begin{array}{l}548.4^{\mathrm{a}} \pm 2.4 \\
\quad(1096)\end{array}$ & $\begin{array}{c}922.9^{\mathrm{a}} \pm 3.7 \\
(890)\end{array}$ & $\begin{array}{c}1225.0^{\mathrm{a}} \pm 4.9 \\
(756)\end{array}$ & - \\
\hline $\begin{array}{l}\text { Overall } \\
\text { mean }\end{array}$ & & $\begin{array}{c}466.3 \pm 1.1 \\
(5442)\end{array}$ & $\begin{array}{c}825.9 \pm 2.2 \\
(3309)\end{array}$ & $\begin{array}{c}1133.9 \pm 3.1 \\
(2729)\end{array}$ & $\begin{array}{c}1301.5 \pm 5.1 \\
(1262)\end{array}$ \\
\hline $\begin{array}{l}\mathrm{R}^{2} \text { of the } \\
\text { model }\end{array}$ & & 0.974 & 0.984 & 0.986 & 0.985 \\
\hline
\end{tabular}

\section{Reproductive traits}

Performance of experimental birds is presented in Table 3. Dead in germ percentage did not vary significantly $(p>0.05)$ among genotypes and in different generations of selection. However, dead in shell percentage significantly $(p<0.05)$ varied among genotypes. Fertility was not significantly different $(\mathrm{p}>0.05)$ among different genotypes but significantly different $(p<0.001)$ among the generations. Hatchability differed significantly $(\mathrm{p}<0.001)$ among the genotypes and generations where $\mathrm{NN}$ had the lowest hatchability. 
Table 2. Variation of body weight gain (least square mean \pm standard error) in different sexes, genotypes and generation of selection

\begin{tabular}{|c|c|c|c|c|c|}
\hline Factor & Level & $\begin{array}{c}\text { 0-8 week } \\
\text { growth }(\mathrm{g} / \mathrm{b} / \\
\text { d) }\end{array}$ & $\begin{array}{c}0-12 \text { week } \\
\text { growth }(\mathrm{g} / \mathrm{b} / \mathrm{d})\end{array}$ & $\begin{array}{c}\text { 0-16 week } \\
\text { growth }(\mathrm{g} / \mathrm{b} / \mathrm{d})\end{array}$ & $\begin{array}{c}\text { Growth at } \\
\text { maturity } \\
(\mathrm{g} / \mathrm{b} / \mathrm{d})\end{array}$ \\
\hline \multirow[t]{2}{*}{ Sex } & Male & $\begin{array}{c}8.5^{\mathrm{a}} \pm 0.03 \\
(2551)\end{array}$ & $\begin{array}{c}10.7^{\mathrm{a}} \pm 0.04 \\
(1249)\end{array}$ & $\begin{array}{c}11.4^{\mathrm{a}} \pm 0.04 \\
(854)\end{array}$ & - \\
\hline & Female & $\begin{array}{c}7.0^{\mathrm{b}} \pm 0.02 \\
(2891)\end{array}$ & $\begin{array}{c}8.1^{\mathrm{b}} \pm 0.03 \\
(2060)\end{array}$ & $\begin{array}{c}8.27^{\mathrm{b}} \pm 0.02 \\
(1875)\end{array}$ & - \\
\hline \multirow[t]{3}{*}{ Genotype } & ND & $\begin{array}{c}7.3^{\mathrm{b}} \pm 0.02 \\
(2910)\end{array}$ & $\begin{array}{c}8.9^{\mathrm{b}} \pm 0.03 \\
(1633)\end{array}$ & $\begin{array}{c}9.3^{\mathrm{b}} \pm 0.03 \\
(1313)\end{array}$ & $\begin{array}{c}7.6^{\mathrm{b}} \pm 0.04 \\
(614)\end{array}$ \\
\hline & DH & $\begin{array}{c}8.6^{\mathrm{a}} \pm 0.03 \\
(1363)\end{array}$ & $\begin{array}{c}10.6^{\mathrm{a}} \pm 0.04 \\
(886)\end{array}$ & $\begin{array}{c}11.1^{\mathrm{a}} \pm 0.04 \\
(728)\end{array}$ & $\begin{array}{c}9.5^{\mathrm{a}} \pm 0.06 \\
(308)\end{array}$ \\
\hline & $\mathrm{NH}$ & $\begin{array}{c}7.3^{\mathrm{b}} \pm 0.04 \\
(1169)\end{array}$ & $\begin{array}{c}8.8^{\mathrm{b}} \pm 0.05 \\
\quad(790)\end{array}$ & $\begin{array}{c}9.0^{\mathrm{c}} \pm 0.05 \\
(688)\end{array}$ & $\begin{array}{c}7.4^{\mathrm{c}} \pm 0.06 \\
(340)\end{array}$ \\
\hline \multirow[t]{4}{*}{ Generation } & $\mathrm{G}_{0}$ & $\begin{array}{c}5.8^{\mathrm{c}} \pm 0.04 \\
(1447)\end{array}$ & $\begin{array}{c}8.8^{c} \pm 0.05 \\
(662)\end{array}$ & $\begin{array}{c}9.0^{\mathrm{d}} \pm 0.05 \\
(660)\end{array}$ & $\begin{array}{c}7.7^{c} \pm 0.05 \\
(429)\end{array}$ \\
\hline & $\mathrm{G}_{1}$ & $\begin{array}{c}7.8^{\mathrm{b}} \pm 0.04 \\
(1328)\end{array}$ & $\begin{array}{l}9.2^{\mathrm{b}} \pm 0.05 \\
(962)\end{array}$ & $\begin{array}{c}9.7^{\mathrm{c}} \pm 0.06 \\
(610)\end{array}$ & $\begin{array}{c}8.6^{\mathrm{a}} \pm 0.05 \\
(421)\end{array}$ \\
\hline & $\mathrm{G}_{2}$ & $\begin{array}{c}8.2^{\mathrm{b}} \pm 0.04 \\
(1571)\end{array}$ & $\begin{array}{c}9.4^{b} \pm 0.05 \\
(795)\end{array}$ & $\begin{array}{c}10.0^{\mathrm{b}} \pm 0.05 \\
(703)\end{array}$ & $\begin{array}{c}8.3^{b} 0.06 \\
(412)\end{array}$ \\
\hline & $\mathrm{G}_{3}$ & $\begin{array}{c}9.2^{\mathrm{a}} \pm 0.04 \\
(1096)\end{array}$ & $\begin{array}{c}10.6^{\mathrm{a}} \pm 0.04 \\
(890)\end{array}$ & $\begin{array}{c}10.6^{\mathrm{a}} \pm 0.04 \\
(756)\end{array}$ & - \\
\hline $\begin{array}{l}\text { Overall } \\
\text { mean }\end{array}$ & & $\begin{array}{c}7.8 \pm 0.02 \\
(5442)\end{array}$ & $\begin{array}{c}9.4 \pm 0.02 \\
(3309)\end{array}$ & $\begin{array}{c}9.8 \pm 0.02 \\
(2729)\end{array}$ & $\begin{array}{c}8.2 \pm 0.03 \\
(1262)\end{array}$ \\
\hline $\begin{array}{l}\mathrm{R}^{2} \text { of the } \\
\text { model }\end{array}$ & & 0.971 & 0.983 & 0.985 & 0.985 \\
\hline
\end{tabular}

Least squares means without a common superscript along the column within a factor differed significantly $(\mathrm{p}<0.05)$; Figure in the parenthesis indicate the number of observations.

The dead in germ and dead in shell percentage of three indigenous chicken types are in agreement with the findings of Khatun et al. (2005) who reported $1.6 \%$ dead in germ and 12.2 dead in shell. Islam et al. (1981) reported that the fertility of upgraded indigenous chicken was $83 \%$, which was lower than that observed in the present study. Khatun et al. (2005) reported that the fertility was $94.8 \%, 88.4 \%$ and $88.1 \%$, respectively in ND, $\mathrm{H}$ and NN genotypes, and was higher than the present findings. Salah Uddin et al. (1995) and Islam et al. (1981) have shown that higher fertility of Bangladeshi local chickens when compared with exotic breeds. The lowest hatchability reported in NN genotype (77.5\%) could be due to the highest egg shell thickness and stronger breaking strength of NN eggs. Khatun et al. (2005) showed that the hatchability ranged from 88.0 to $94.9 \%$ in different genotypes with the overall percentage of 86 , which was slightly higher than the present findings. The age at first lays ranged from 150.0 to 159.5 days. The age at first lay reported in the present study was comparable to same indigenous chicken genotypes as reported by Faruque et al. (2007). 
Table 3. Variation of reproductive performance (least square mean \pm standard error) in different sexes, genotypes and generation of selection

\begin{tabular}{|c|c|c|c|c|c|c|}
\hline Factor & Level & $\begin{array}{c}\text { Dead in } \\
\text { germ } \\
(\%)\end{array}$ & $\begin{array}{c}\text { Dead in } \\
\text { shell }(\%)\end{array}$ & $\begin{array}{c}\text { Fertility } \\
(\%)\end{array}$ & $\begin{array}{c}\text { Hatchabili } \\
\text { ty }(\%)\end{array}$ & $\begin{array}{c}\text { AFE } \\
\text { (days) }\end{array}$ \\
\hline \multirow[t]{3}{*}{ Genotype } & ND & $\begin{array}{c}2.2^{\mathrm{a}} \pm 0.4 \\
(575)\end{array}$ & $\begin{array}{c}8.2^{\mathrm{b}} \pm 0.9 \\
(555)\end{array}$ & $\begin{array}{c}85.7^{\mathrm{a}} \pm 1.0 \\
(575)\end{array}$ & $\begin{array}{c}89.0^{\mathrm{a}} \pm 1.0 \\
(555)\end{array}$ & $\begin{array}{c}155.0^{\mathrm{b}} \pm 0.6 \\
(376)\end{array}$ \\
\hline & $\mathrm{DH}$ & $\begin{array}{c}2.3^{\mathrm{a}} \pm 0.5 \\
(302)\end{array}$ & $\begin{array}{c}10.0^{\mathrm{b}} \pm 1.2 \\
(288)\end{array}$ & $\begin{array}{c}85.2 \pm 1.4 \\
(302)\end{array}$ & $\begin{array}{c}85.6^{\mathrm{b}} \pm 1.4 \\
(288)\end{array}$ & $\begin{array}{c}159.5^{\mathrm{a}} \pm 0.7 \\
(200)\end{array}$ \\
\hline & $\mathrm{NN}$ & $\begin{array}{c}3.1^{\mathrm{a}} \pm 0.5 \\
(300)\end{array}$ & $\begin{array}{c}19.8^{\mathrm{a}} \pm 1.3 \\
(292)\end{array}$ & $\begin{array}{c}85.4^{\mathrm{a}} \pm 1.4 \\
\quad(300)\end{array}$ & $\begin{array}{c}77.5^{\mathrm{c}} \pm 1.4 \\
(292)\end{array}$ & $\begin{array}{c}152.7^{\mathrm{b}} \pm 0.9 \\
(205)\end{array}$ \\
\hline \multirow[t]{3}{*}{ Generation } & $\mathrm{G}_{0}$ & $\begin{array}{c}2.3 \pm 0.5 \\
(308)\end{array}$ & $\begin{array}{c}11.0 \pm 1.2 \\
(300)\end{array}$ & $\begin{array}{c}82.5^{\mathrm{b}} \pm 1.4 \\
(308)\end{array}$ & $\begin{array}{c}87.5^{\mathrm{a}} \pm 1.4 \\
(300)\end{array}$ & $\begin{array}{c}161.5^{\mathrm{b}} \pm 0.5 \\
\quad(391)\end{array}$ \\
\hline & $\mathrm{G}_{1}$ & $\begin{array}{c}3.4 \pm 0.4 \\
(533)\end{array}$ & $\begin{array}{c}12.5 \pm 1.0 \\
(504)\end{array}$ & $\begin{array}{c}84.6^{\mathrm{b}} \pm 1.1 \\
(533)\end{array}$ & $\begin{array}{c}84.7^{\mathrm{a}} \pm 1.1 \\
(504)\end{array}$ & - \\
\hline & $\mathrm{G}_{2}$ & $\begin{array}{c}2.1 \pm 0.5 \\
(336)\end{array}$ & $\begin{array}{c}15.8 \pm 1.2 \\
(331)\end{array}$ & $\begin{array}{c}90.2^{\mathrm{a}} \pm 1.3 \\
(336)\end{array}$ & $\begin{array}{c}78.0^{\mathrm{b}} \pm 1.3 \\
(331)\end{array}$ & $\begin{array}{c}150.0^{\mathrm{a}} \pm 0.6 \\
(390)\end{array}$ \\
\hline $\begin{array}{l}\text { Overall } \\
\text { mean }\end{array}$ & & $\begin{array}{c}2.6 \pm 0.3 \\
(1177)\end{array}$ & $\begin{array}{c}12.9 \pm 0.7 \\
(1135)\end{array}$ & $\begin{array}{c}85.4 \pm 0.7 \\
(1177)\end{array}$ & $\begin{array}{c}83.8 \pm 0.7 \\
(1135)\end{array}$ & $\begin{array}{c}155.7 \pm 0.4 \\
(781)\end{array}$ \\
\hline $\begin{array}{l}\mathrm{R}^{2} \text { of the } \\
\text { model }\end{array}$ & & 0.982 & 0.953 & 0.928 & 0.930 & 0.995 \\
\hline
\end{tabular}

Least squares means without a common superscript along the column within a factor differed significantly $(\mathrm{p}<0.05)$; Figure in the parenthesis indicate the number of observations.

\section{Egg production traits}

The effects of genotype, generation of selection and their interactions on egg production traits are presented in Tables 4 and 5. The 242 day and 315 day egg productions and respective egg production rates were influenced by genotype and generation (Table 4). Among three indigenous genotypes, ND produced significantly $(\mathrm{p}<0.001)$ highest number of eggs whereas $G_{2}$ birds were with significantly $(p<0.001)$ best egg production (Table 4$)$. However, some studies have reported that naked neck $(N N)$ laid a significantly $(p<0.05)$ higher number of eggs compared to frizzle and normal feathered chicken types Yoshimura et al. (1997), Moreki and Mosupu (2003). Petersen et al. (1991) reported that the village chicken produced an average of 100 eggs per year whereas the egg production of the Ethiopian (Tadelle et al. 2003) and Bangladeshi (Huque and Haque, 1990) village chicken were reported to be 75 and 35-45 eggs per year, respectively. Bhuiyan et al. (2005) revealed that annual egg production per hen was 50-55 in NN and 45-50 in indigenous chicken under scavenging. Huque (1999) found the annual egg production of selected NN, H and ND was 141, 101 and 121 respectively under intensive rearing which is in agreement with present findings.

Hen-day egg production (HDEP \%) and hen-house egg production (HHEP \%) of the present study were affected significantly $(\mathrm{p}<0.001)$ by genotype, generation and genotype $\times$ generation interaction (Table 5). It was observed that HDEP\% and HHEP\% were high in $\mathrm{G}_{2}$ due to selection. Significant effect among different genotypes on HDEP\% and HHEP\% found in this study confirms the previous report by Miah et al. (2002) and Faruque et al. (2010) found that breed had significant $(\mathrm{p}<0.05)$ effect on hen-day egg production. In this study the highest HDEP\% and HHEP\% were found in ND (51.4 \pm 0.4 and $49.7 \pm 0.4)$. 
Table 4. Variation of egg production (least square mean \pm standard error) in different sexes, genotypes and generation of selection

\begin{tabular}{|c|c|c|c|c|c|}
\hline Factor & Level & $\begin{array}{l}242 \text { days EP } \\
\text { (no.) }\end{array}$ & $\begin{array}{c}242 \text { days EP rate } \\
(\%)\end{array}$ & $\begin{array}{c}315 \text { days EP } \\
\text { (no.) }\end{array}$ & $\begin{array}{c}315 \text { days } \\
\text { EP rate } \\
(\%)\end{array}$ \\
\hline \multirow[t]{3}{*}{ Genotype } & ND & $\begin{array}{c}51.7^{\mathrm{a}} \pm 0.5 \\
(584)\end{array}$ & $\begin{array}{c}58.7^{\mathrm{a}} \pm 0.5 \\
(584)\end{array}$ & $\begin{array}{c}83.9^{\mathrm{a}} \pm 1.1 \\
(388)\end{array}$ & $\begin{array}{c}52.1^{\mathrm{a}} \pm 0.6 \\
(388)\end{array}$ \\
\hline & $\mathrm{H}$ & $\begin{array}{c}43.2^{c} \pm 0.7 \\
(291)\end{array}$ & $\begin{array}{c}49.1^{\mathrm{c}} \pm 0.8 \\
(291)\end{array}$ & $\begin{array}{c}76.2^{\mathrm{b}} \pm 1.4 \\
(208)\end{array}$ & $\begin{array}{c}47.3^{\mathrm{b}} \pm 0.8 \\
(208)\end{array}$ \\
\hline & $\mathrm{NN}$ & $\begin{array}{c}45.4^{\mathrm{b}} \pm 0.9 \\
\quad(293)\end{array}$ & $\begin{array}{c}51.6^{\mathrm{b}} \pm 1.0 \\
(293)\end{array}$ & $\begin{array}{c}74.0^{\mathrm{b}} \pm 1.5 \\
\quad(209)\end{array}$ & $\begin{array}{c}46.0^{\mathrm{b}} \pm 0.9 \\
\quad(209)\end{array}$ \\
\hline \multirow[t]{3}{*}{ Generation } & $\mathrm{G}_{0}$ & $\begin{array}{c}43.8^{c} \pm 0.6 \\
\quad(396)\end{array}$ & $\begin{array}{c}49.7^{\mathrm{c}} \pm 0.7 \\
\quad(396)\end{array}$ & $\begin{array}{c}74.9^{\mathrm{b}} \pm 1.0 \\
(399)\end{array}$ & $\begin{array}{l}46.5^{\mathrm{b}} \pm 0.6 \\
\quad(399)\end{array}$ \\
\hline & $\mathrm{G}_{1}$ & $\begin{array}{c}45.3^{\mathrm{b}} \pm 0.7 \\
\quad(387)\end{array}$ & $\begin{array}{c}51.4^{\mathrm{b}} \pm 0.7 \\
(385)\end{array}$ & - & - \\
\hline & $\mathrm{G}_{2}$ & $\begin{array}{c}50.9^{\mathrm{a}} \pm 0.9 \\
(385)\end{array}$ & $\begin{array}{c}57.9^{\mathrm{a}} \pm 1.1 \\
(385)\end{array}$ & $\begin{array}{c}81.2^{\mathrm{a}} \pm 1.1 \\
\quad(406)\end{array}$ & $\begin{array}{c}50.4^{\mathrm{a}} \pm 0.7 \\
\quad(406)\end{array}$ \\
\hline Overall mean & & $\begin{array}{c}46.5 \pm 0.4 \\
(1168)\end{array}$ & $\begin{array}{c}52.9 \pm 0.5 \\
(1168)\end{array}$ & $\begin{array}{c}78.0 \pm 0.7 \\
(805)\end{array}$ & $\begin{array}{l}48.5 \pm 0.4 \\
\quad(805)\end{array}$ \\
\hline $\begin{array}{l}\mathrm{R}^{2} \text { of the } \\
\text { model }\end{array}$ & & 0.944 & 0.944 & 0.945 & 0.945 \\
\hline
\end{tabular}

Table 5. Variation of hen house (HHEP) and hen day egg production (HDEP) (least square mean \pm standard error) in different sexes, genotypes and generation of selection

\begin{tabular}{llcc}
\hline Factor & & HDEP (\%) & HHEP (\%) \\
\hline Genotype & $\mathrm{ND}$ & $51.4^{\mathrm{a}} \pm 0.4(324)$ & $49.7^{\mathrm{a}} \pm 0.4(324)$ \\
& $\mathrm{H}$ & $44.0^{\mathrm{c}} \pm 0.4(324)$ & $41.7^{\mathrm{b}} \pm 0.4(324)$ \\
& $\mathrm{NN}$ & $48.1^{\mathrm{b}} \pm 0.4(324)$ & $42.8^{\mathrm{b}} \pm 0.4(324)$ \\
Generation & $\mathrm{G}_{0}$ & $44.6^{\mathrm{b}} \pm 0.3(486)$ & $41.4^{\mathrm{b}} \pm 0.3(486)$ \\
& $\mathrm{G}_{1}$ & - & - \\
& $\mathrm{G}_{2}$ & $51.0^{\mathrm{a}} \pm 0.3(486)$ & $48.1^{\mathrm{a}} \pm 0.3(486)$ \\
Overall mean & & $47.8 \pm 0.2(972)$ & $44.7 \pm 0.2(972)$ \\
$\mathrm{R}^{2}$ of the model & & 0.970 & 0.967 \\
\hline
\end{tabular}

Least squares means without a common superscript along the column within a factor differed significantly $(\mathrm{p}<0.05)$; Figure in the parenthesis indicate the number of observations.

\section{Response to selection}

Both male and female birds showed a considerable response to selection as observed in 8 weeks body weight (Table 6). As estimated, the expected response to selection of 8 weeks body weight was highest in $\mathrm{H}$ (53.98 g vs. $24.70 \mathrm{~g})$ males and females compared to other genotypes. 
Table 6. Expected response to selection in 8 weeks body weight (g) of indigenous chicken

\begin{tabular}{ccccc}
\hline Genotype & Sex & Response in $\mathbf{G}_{\mathbf{1}}$ & Response in $\mathbf{G}_{\mathbf{2}}$ & Average response \\
\hline \multirow{2}{*}{ ND } & M & 57.91 & 49.26 & 52.03 \\
& F & 11.28 & 23.60 & 20.92 \\
\multirow{2}{*}{ H } & M & 81.72 & 28.56 & 53.98 \\
& F & 34.30 & 12.34 & 24.70 \\
NN & M & 52.90 & 17.03 & 37.40 \\
& F & 14.89 & 7.26 & 12.43 \\
\hline
\end{tabular}

Table 7 shows the expected selection responses for egg production to 280 days in $G_{0}$ and $G_{2}$ generations of three genotypes of chicken. The observed response in egg number over generations was $0.7 \%$ which was less than expected response.

The results indicated that the genetic improvement for egg production will be effective in selection and breeding program. It could be seen that the realized genetic gain in different genotypes was different from prediction. The possible reason for variable responses among genotypes could either be genotype and environment interaction or correlated responses. Another factor that may have contributed to the variability of response from generation to generation may also be due to varying seasons of hatching across generations.

Table 7. Expected response to selection for egg production (\%) up to 280 days

\begin{tabular}{cccc}
\hline Genotype & Response in $\mathbf{G}_{\mathbf{1}}$ & Response in $\mathbf{G}_{\mathbf{2}}$ & $\begin{array}{c}\text { Observed } \\
\text { response }\end{array}$ \\
\hline ND & 1.83 & 2.28 & 0.722 \\
H & 3.10 & 3.28 & \\
NN & 2.46 & 3.59 & \\
\hline
\end{tabular}

As seen in Table 8, the responses to selection for egg weight at 40 weeks in ND were expected to be negative in $G_{1}$ and positive in $G_{2}$. The responses to selection for egg weight at 40 weeks in $\mathrm{H}$ were expected to be negative in both $\mathrm{G}_{1}$ and $\mathrm{G}_{2}$ generation. On the other hand, the responses to selection for egg weight at 40 weeks in NN were expected to be positive in both $\mathrm{G}_{1}$ and $\mathrm{G}_{2}$ generation. Nwagu et al. (2007) in their study estimated genetic change of average egg weight in female and male Rhode Island chicken to be -0.89 and $-0.43 \mathrm{~g}$, respectively per generation which partially agrees with the results of this study (-0.01 to 0.68 $\mathrm{g}$ after two generations). The results imply that the genetic improvement for the trait is possible only for $\mathrm{NN}$ genotype of chicken.

Table 8. Expected response to selection for egg weight (g) at 40 week

\begin{tabular}{ccc}
\hline Genotype & Response in $\mathbf{G}_{\mathbf{1}}$ & Response in $\mathbf{G}_{\mathbf{2}}$ \\
\hline ND & -0.07 & 0.10 \\
H & -0.05 & -0.01 \\
NN & 0.01 & 0.68 \\
\hline
\end{tabular}

*ND=Non-descript Deshi; H=Hilly; $\mathrm{NN}=$ Naked Neck; $\mathrm{G}_{0}=$ Foundation stock; $\mathrm{G}_{2}=$ Second generation 


\section{CONCLUSION}

Non-descript Deshi chicken has a potential in improving for egg production purposes whereas Hilly chicken will be suitable for meat purposes. Reproductive performance of Nondescript Deshi chicken is comparatively better than those of other two genotypes. The study of responses to selection in progressive generations revealed that genetic gain by selection of birds on the basis of 8 weeks body weight will be effective for Hilly and Naked Neck genotypes, although there appears a decreasing trend of genetic gain. Response to selection for egg production trait will be effective for Non-descript Deshi and Naked Neck. Selection of birds on the basis of egg weight at 40 weeks will not be effective for Non-descript Deshi and Hilly genotype, although it will be effective only for Naked Neck genotype.

\section{ACKNOWLEDGEMENTS}

Authors are thankful to the UNEP-GEF-ILRI-FAnGR Asia Project for promoting this work and financial support of first author's participation at $26^{\text {th }}$ PGIA Annual Congress, Sri Lanka.

\section{REFERENCES}

Bhuiyan, A.K.F.H., Bhuiyan, M.S.A. and Deb, G.K. (2005). Indigenous Chicken genetic resources in Bangladesh: Current status and future outlook. Animal Genetic Resources Information's Bulletin., 36, 73 - 84.

Bhuiyan, A.K.F.H., Biswas, S.R. and Biswas, J.C. (2009). Genetic dilution of indigenous chicken in selected villages of Bangladesh. Proceedings of the Sixth International Poultry Show and Seminar, WPSA-BB, 5-7 March 2009, Dhaka, Bangladesh, pp. 147 - 162.

Crawford, R.D. (1984). Assessment and conservation of animal genetic resources in Canada. Canadian J. Anim. Sci., 64, 235 - 251.

Falconer, D.S. (1981). Introduction to Quantitative Genetics. Second Edition. Chapter 11. Selection response and its prediction, pp. $171-177$.

Faruque, S., Afroz, M.A. and Islam, M.S. (2010). Evaluation of response to selection in chicken. Int. J. BioRes., 1(4), 01 - 05.

Faruque, S., Rahman, M.M. and Islam, M.N. (2011). Evaluation of the performance of native chickens and estimation of genetic parameter for body weight. Proceedings of the Seventh International Poultry Show and Seminar, WPSA-BB, 25-27 March 2011, Dhaka Bangladesh, pp. 199-206.

Faruque, S., Sarker, N.R., Islam, M.N. and Sarker, M.S.K. (2007). Performance of native chickens under intensive system. J. Bangladesh Agril. Univ., 5(2), 283 - 288.

Halima, H. (2007). Phenotypic and genetic characterization of indigenous chicken populations in Northwest Ethiopia. Thesis submitted to the Faculty of Natural and Agricultural Sciences, Department of Animal, Wildlife and Grassland Sciences, University of the Free State, Bloemfontein, South Africa. 
Huque, Q.M.E. (1999). Poultry Research and Development in Bangladesh. Proceedings of the $1^{\text {st }}$ Seminar and International Poultry show, April 24-26, IDB Bhaban, Sher-e- Bangla Nagar, Dhaka Bangladesh, pp. 70-80.

Huque, Q.M.E. and Hauqe, M.E. (1990). The onset of lay in indigenous hens following hatching chicks. Poultry Adviser, 13, 57 - 60.

Islam, A.B.M.M., Huque, M.M. and Rahim, Q.M.F. (1981). Reproductive performance of upgraded indigenous chicken. Poultry Adviser, 14, 32 - 37.

Khandoker, M.A.M.Y. (1993). Performance of Indigenous (Deshi), Rhode Island Red (RIR) and Deshi $\times$ RIR chickens under farm condition. Master of Science in Poultry Science, Thesis, submitted to the department of Poultry Science, Bangladesh Agricultural University, Mymensingh.

Khatun, R., Islam, M.S., Faruque, S., Azmal, S.A. and Uddin, M.S. (2005). Study on the productive and reproductive performance of 3 native genotypes of chicken under intensive management. J. Bangladesh Agril. Univ., 3, 99 - 104.

Kramer, C.Y. (1957). Extension of Multiple Range Tests to Group Correlated Adjusted Means. Biomet., 13(1), 13 - 18.

Miah, M.S., Islam, M.A. and Ali, M.A. (2002). Growth and egg production performance of exotic pure breeds and crossbreeds chicken. Bangl. Vet., 19 (1), 43 - 47.

Moreki, J.C. and MD Masupu, P.K. (2003). Country reported on local chicken production: Bostwana. J. Poult. Sci., 24 (4), 407 - 420.

Nwagu, B.I., Olorunju, S.A.S., Oni, O.O., Eduvie, L.O., Adeyinka, I.A., Sekoni, A.A. and Abeke, F.O. (2007). Response of egg number to selection in Rhode Island Red Chickens selected for part period egg production. International J. Poult. Sci., 6(1), 18 - 22.

Petersen, J.B., Guzman, Jr. M.R.D., and Wu, M.C. (1991). Catalog of the native Poultry of Southeast Asia Food and Fertilizer Technology Centre for the Asian and Pacific Region. Taiwan Livestock Research Institute, Taiwan.

Salah Uddin, M., Yeasmin, T. and Howlider, M.A.R. (1995). Relationship between fertility and hatchability with egg weight of free-range native chicken. Bang. J. Train. Dev., 8 (192), $99-102$.

Singh, B.P., Chaudhary, R.P., Singh, R.V. and Ahlawates, S.P.S. (1982). Diallel crosses in poultry for broiler production, estimation of heterosis for various broiler traits. Ind. J., 59, $882-892$.

Snedecor, G.W. and Cochran, W.G. (1989). Statistical Methods, 8th edn. The Iowa State University Press, Ames., IA, USA.

SPSS, (1998). SPSS Base 11.5 Application Guide, SPSS Inc., USA.

Tadelle, D., Million, T., Alemu, Y. and Peters, K.J. (2003). Village chicken production system in Ethiopia:1. Flock characteristics and performance. Livest. Res. Rural Dev., 15(1). 
Wilson, R.T., Traore, A., Traore, A., Kuit, H.G. and Slingerland, M. (1987). Livestock Production in Central Mali: Reproduction, growth and mortality of domestic fowl under traditional management. Trop. Anim. Hlth. Prod., 19, 229 - 236.

Yoshimura, Y., Barua, A., Heryanto, B., Ohira, H. and Zheng, W. (1997). Reproductive physiology in domestic animal as a basic knowledge to improve poultry production in Asian countries. J. Agril. Sci., 41, 367 - 370. 